\title{
PROPERTIES AND MECHANISMS OF ACTION OF NON- REPLICATIVE VIRAL VECTORS, INACTIVATED VIRUSES, RNA AND DNA VACCINES
}

\begin{abstract}
Alejandro Durán-Méndez ${ }^{1,3}$, Miranda Tobón-Cubillos ${ }^{1,2}$, América Viveros-Hernández $z^{1,3}$, Jorge Rafael Flores-Hernández $z^{1,2}$, Juan Daniel Méndez-Coca ${ }^{1,2}$, Alberto N. Peón ${ }^{1,4, *}$

${ }^{1}$ Laboratorio Santiago Ramón y Cajal, Sociedad Española de Beneficencia, Pachuca, Hidalgo; Av. Juárez \#908, Col. La Villita, Pachuca, Hidalgo, México, CP42060.

2 Facultad de Medicina, Benemérita Universidad Autónoma de Puebla, México.

${ }^{3}$ Área Académica de Medicina, Universidad Autónoma del Estado de Hidalgo.

4 Escuela Superior de Apan, Universidad Autónoma del Estado de Hidalgo. Carretera Apan-Calpulalpan s/n, Colonia 43920 Chimalpa Tlalayote, Hgo, México.
\end{abstract}

\section{Abstract}

In the last 40 years new vaccine technologies have arisen, promising easy and quick formulation and design. In the scenario of a rapidly-spreading infectious pathogen, these technologies may render an adequate strategy to counter the pandemic. As such, me review the engineering and mechanisms of action of the main anti-COVID-19 vaccines.

\section{Highlights}

Anti-COVID-19 RNA vaccines were formulated 60 days after the SARSCoV-2 genome was sequenced.

Non-replicative viral vectors have shown a durable immunity and good efficacy.

\section{Keywords}

COVID-19

Vaccines

SARS-COV-2

RNA vaccines

DNA vaccines

Correspondence should be addressed to: investigacion@benepachuca.com 
SÖC̊IIÉÉÁAD

ESPAÑOLA BENEFICENCIAAC

P $A$ A $C$ C
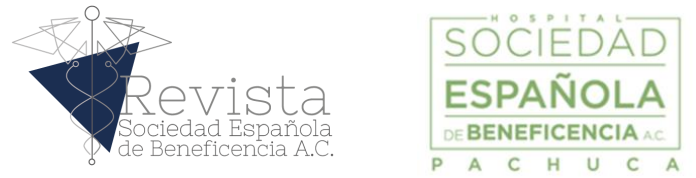
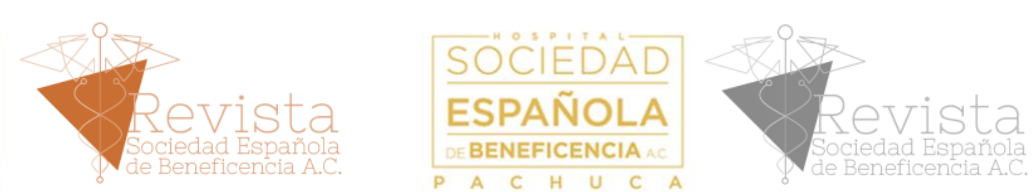

\section{Introduction}

Infectious diseases posed the greatest threats to public health and were the cause of uncountable deaths. This began to change during the last 150 years, since microbes were better understood, and vaccines, antiviral and antibacterial compounds were discovered, allowing many ancient diseases such as smallpox, polio and measles, to be explained and controlled.1.

The control of these diseases happened because of vaccination programs. Vaccination is one of the greatest achievements of public health ${ }^{2,3}$, as it has made vaccine-preventable diseases a concern from the past. With this knowledge, the past decades have witnessed the development of a wide array of new vaccination technologies ranging from targeted attenuation techniques of live pathogens to the delivery of biologically engineered protein and peptide antigens as well as viral vector and nucleic acid-based antigens ${ }^{4}$.

On the other hand, in a century where the main public health conflicts are due to chronic-degenerative diseases, the emergence of the SARS-CoV-2 virus has represented one of the main global threats today, and fastly, we are seeing the development and distribution of anti-SARS-CoV-2 vaccines by different countries and companies, that are using many of these different technologies 5,6 as a response to the pandemic. In this article we explain the properties and mechanisms of action of non-replicative viral vectors, inactivated viruses, RNA and DNA vaccines, as well as the degrees of development that each vaccine has.

\section{DIVERSITY IN VACCINE FORMULATIONS: A BIOTECHNOLOGICAL RESPONSE TO VARIED PATHOGENS}

Vaccination is a powerful strategy to counter infectious diseases. In the absence of pre-existing protective immunity, potentially novel or emerging pathogens may cause emerging pandemics?. But usually, vaccine development involves 5 to 10 years to get approval, in the best scenarios, which is not fast enough to hinder fast-spreading pathogens, like SARS-CoV-2. In the process of approval, it is necessary that the vaccine passes through three phases of clinical trials and ultimately, through regulatory approval ${ }^{8,9}$.

Traditional vaccine development using attenuated or inactivated pathogens allowed the successful reduction of infectious diseases in the past, and even the near eradication of some others. Nevertheless, these methods might not be optimal in emergency situations due to the risk of reversion, the undesired effects, or the time-consuming process that is required for their production ${ }^{4}$.

In the context of a pandemic, a low-cost and scalable manufacturing processes are required for global availability, as is the case of nucleic acid vaccines ${ }^{7}$. As a suitable option for emerging diseases, viral vector-based vaccines or nucleic acid-vaccine technologies came out, with nucleic acid-based vaccines needing shorter times for development, manufacturing and production ${ }^{10}$. In this way, only 
SÖC̊IIÉÉÁAD

ESPAÑOLA BENEFICENCIA $A C$

$P$ A C H U C A

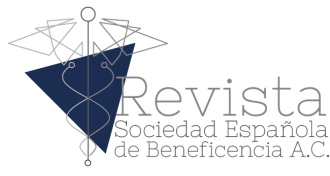

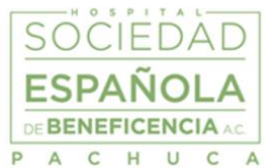

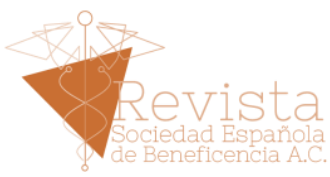

66 days since SARS-CoV-2 genome was sequenced an mRNA vaccine started human testing'. RNA vaccines are not an entirely new development, as they were initially developed 30 years ag ${ }^{11}$, nonetheless, not a single RNA vaccine has hit the market until now because pharmaceutical companies did not considered such formulations as good options because the cold chains needed to distribute them was lacking in the entire world ${ }^{12}$.

The use of new vaccination technologies for emergency situations represents a potential solution to other infectious diseases, leading a fast and effective approach for the rest of pathogens.

\section{NON-REPLICATIVE VIRAL VECTORS}

The viral vectors are defined as non-pathogenic viral vehicles into which immunodominant antigen genes from microbial pathogens are transfected, as a means to cause their expression and ultimately their recognition by the host's immune system $^{13}$. Because of the display of the antigens onto a viral vehicle, these vaccines are self-adjuvant and share similar characteristics with viral infections, except that they are inoculated in unusual sites and are no replicative. In general terms, due to their convergences viral vectors can induce similar immune responses as those that happen by naturally-acquired viral infections. Several innate immunity receptors recognize viral structures and trigger the expression of cytokines, which cause cell activation and promote an anti-viral state. Then, an adaptive immune is activated when antigen presenting cells (APCs) interact with T cells. Finally, all these processes lead antibodies production after B cell activation $^{14}$.

Some of the advantages of using this technique is that the characteristics, type, and intensity of the immune response are principally determined by the vector and not the pathogen. On the other hand, in this scenario of the SARS-CoV-2 pandemic, the development and test of this kind of vaccines can be significantly shortened with a great level of safety and efficacy ${ }^{15}$.

Currently, there are three vectors that are most understood and widely used as gene therapy: adenoviral (Ad), lentiviral (LV), and adeno-associated viral vectors $(\mathrm{AAV})^{14}$. Generally, a viral vector has three components: a) the protein capsid and/or envelope, b) the transgene, and c) the combined enhancer, promoter, and auxiliary elements of the transgene. Each of these elements create an intended effect, for example, the capsid defines the tropism of the vaccine and boosts antigen recognition; the transgene induces the clonal proliferation of a select specific lymphocyte population with the subsequent creation of memory $\mathrm{T}$ and $\mathrm{B}$ cells; and finally the different molecular elements help in the establishment of the transgene expression ${ }^{16}$.

Ad vectors are formed by a protein capsid that envelope a 36-kb double-strained DNA genome. This genetic material stays epichromosomal, because critical protease genes for the viral replication are removed to avoid a complete replication. After the injection of the viral vector, activation of vascular endothelial cells and platelets occur, as well as cytokine release (e.g. interleukin [IL] 2, IL-12, IL-15, IL- 
18), which begin to create an ideal inflammatory environment to promote adaptive immunity ${ }^{14}$. Additionally, infected cells at the site of infection (e.g. myocites) express the immunogen and is presented trough the major histocompatibility complex (MHC) class I to CD8 ${ }^{+} \mathrm{T}$ cells, leading cytokine release, CD8 ${ }^{+} \mathrm{T}$ cell degranulation and induction of necrosis and apoptosis. Then, APCs phagocyte necrotic/apoptotic bodies and/or expressed antigens and generate both CD ${ }^{+}$and $\mathrm{CD}^{+} \mathrm{T}$ cell responses ${ }^{17}$, while the expressed antigens are released by necrotic cells (Fig. 1). Due to the latter, it is possible that antibody production happens but it is not as efficient as the B-cell response that the vector itself induces ${ }^{14}$. Nevertheless, the innate immune response at site of administration can inactivate Ad vectors, and high-vaccine doses can be associated to systemic responses that could be lethal ${ }^{18}$.

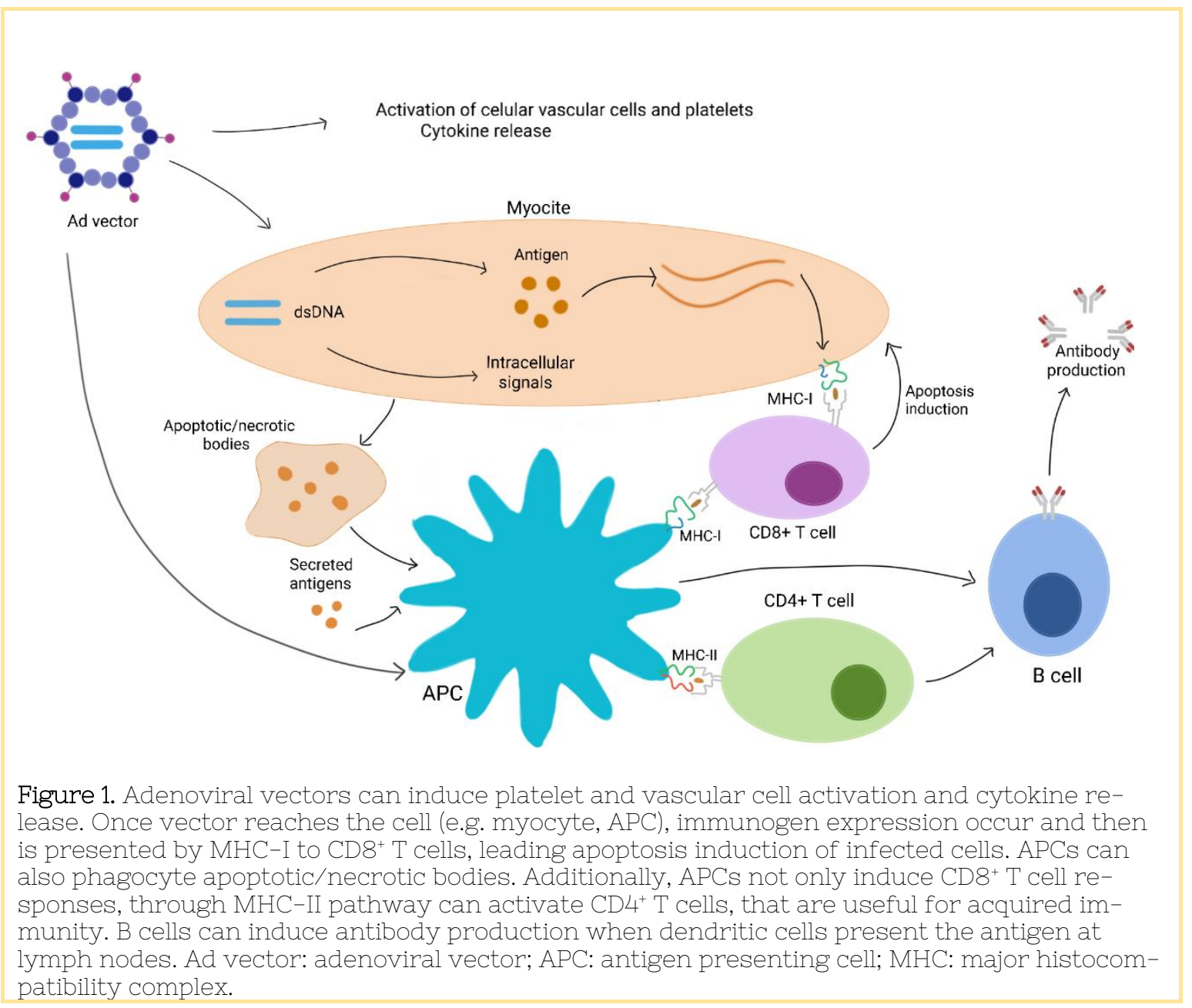

AAV vectors consists in a small non-enveloped parvovirus with a 5-kb singlestrained or 2.5-kb genome that contains no replicating genes, which confers more security ${ }^{14}$. As such, these vectors do not interact directly with host's genome, stay as episomes and confers less genotoxic effects compared to Ad vec- 
SÖC̊IIÉÉÁAD

ESPAÑOLA BENEFICENCIAAC

P $A$ A $C$ C H U C C A

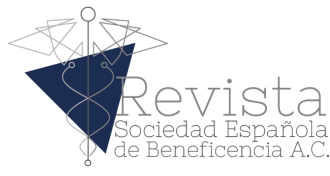

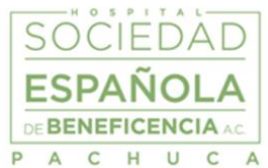

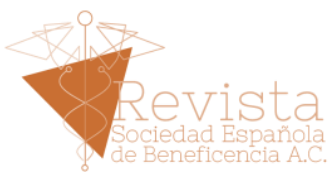

tors $^{18}$. However, innate immunity is transient and weak, and antigen transcription seems to be ineffective. CD8 ${ }^{+} \mathrm{T}$ cell responses are developed mainly through their interaction with plasmacytoid dendritic cells and their TLR9, allowing liberation of cytokines (e.g. IFN- $\gamma$ ). However, conventional DC can interact with the AAV and induce adaptive immunity, but this response occurs in variable degrees and depends on additional characteristics of the vector, such their design or administration route ${ }^{14}$. Nonetheless, transgene expression is longer than that in Ad vectors ${ }^{18}$.

LV contain a single-strained RNA, but being retroviruses, their genetic material is transcribed to DNA in the host cell. Once it reaches the cell's nucleus, the cell can express the transgene for as long as the infected cell persists. The transgene is presented by the MHCI complex, while some of the infected cells eventually die due to the slow lytic cycle of the virus, releasing antigens. Thus, B and T cell responses appear, with an efficient antibody production. Nevertheless, immune responses could be limited by INF- $\alpha$, which is highly expressed as a part of the antiviral response and limits transgene expression ${ }^{14}$. Additionally, the LV genome integration to host's cell raises the possibility of oncogenesis, inducing transgene cells transformation ${ }^{18}$.

\section{INACTIVATED VIRUSES}

These vaccines are produced by the growth of the agent in a culture medium, and they are inactivated by physical or chemical means ${ }^{19}$. There are some different methods to reach inactivation. Formaldehyde, glutaraldehyde, and binary ethylenimine are examples of alkylating and crosslinking agents, that cause the blockage of genome reading as well as the modification and functional inactivation of viral proteins. Lastly, radiation can be also used (e.g. gamma radiation, ultraviolet light) causing direct damage to the genome and structural modifications to viral proteins ${ }^{20}$.

In this instance, pathogens are not killed but they cannot reproduce inside the host. Nonetheless, they maintain their molecular integrity, and thus can be recognized by the immune system to evoke an adaptive immune response ${ }^{21,22}$. The immune response to these vaccines is mainly humoral, but a cellular component is also present22,23, as immunity usually depends upon APCs acting by the MHC class I and II pathways ${ }^{23}$.

As these vaccines activate immunity only through the pathogen-associated molecular patterns (PAMPS) that are natural to the inactivated virus, but no alarm signals are produced during the action of the vaccines, the immunity that these vaccines provide is relatively weak. As a consequence, inactivated-virus vaccines promote immunity after the second or third dose ${ }^{24}$.

These vaccines are more stable, as they are embedded into adjuvants that preserve their structure; and are also considered safer than live vaccines, because non-reproducing microbes cannot mutate back to their disease-causing state, 


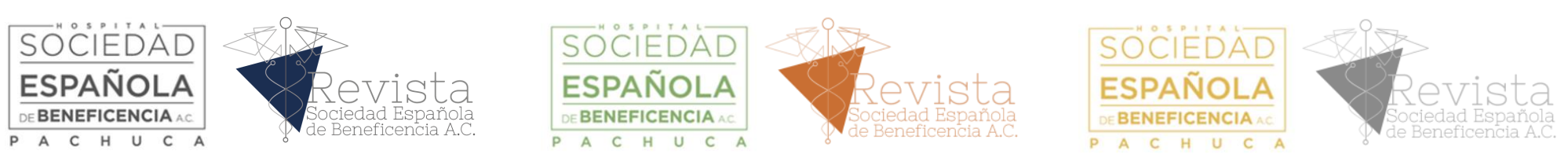

like attenuated vaccines can. Inactivated vaccines generally do not require refrigeration and can be easily stored and transported in lyophilized form, making them more accessible to people in developing countries ${ }^{23}$.

Despite the great advantage of their easy design and formulation, not all the viruses are susceptible to the development of inactivated vaccines. This phenomenon is derived from the fact that the inactivation processes tend to alter antigen's three dimensional, and even two dimensional structures, leading to aberrant immune recognition.

\section{DNA VACCINES}

DNA vaccines are formed by recombinant bacterial plasmids that encode antigens. They have a eukaryotic promoter, which assures proper gene expression and protein synthesis in its native form. DNA vaccine design is straightforward, and it only requires one-step cloning, which allows for massive and fast production, and thus are appropriate tools for emergency situations. These vaccines can be administered by several routes, such as intradermal, intramuscular, transdermal or mucosal, allowing for a more compartimentalized induction of immunity. Moreover, DNA vaccines allow for standard DNA-modifying techiques, which copes with the need of redisigning the formulations of vaccines against pathogens that exhibit constant antigenic drift ${ }^{25}$. The plasmid is also very stable to temperature changes and have less need of refrigeration, allowing an easier transport and use in endemic areas in lyophilized forms; their safety lies in excluding the need of purifying proteins from pathogens ${ }^{26,27}$. Unfortunately, DNA vaccines have shown effective immune protection in animal models, but limited effects in humans ${ }^{26}$, nonetheless, these limitations may be overcome with research about the promoters.

Other elements included in these vaccines are adjuvants that lead to a better immune response. These adjuvants can be elements such as alum, or even cytokines or signaling molecules also encoded by the plasmid25.

These vaccines require the release of genes able to encode antigens using DNA plasmids as a vector ${ }^{27}$. After the administration, the DNA deposed in intracellular spaces needs to reach cell nuclei to lead the transcription of mRNA ${ }^{26}$. This process evolves several factors and pathways. The first step is the endocytosis of the DNA plasmid maybe by an actin-based movement. Then, several proteins bind to the plasmid forming DNA-protein complexes, warranting the movement of this complex in direction to the nucleus through microtubules. The next step is the entry of the DNA plasmid into the nucleus, which is mediated by importins, or directly when mitosis occur (because of the absence of nuclear envelope) ${ }^{28}$. 


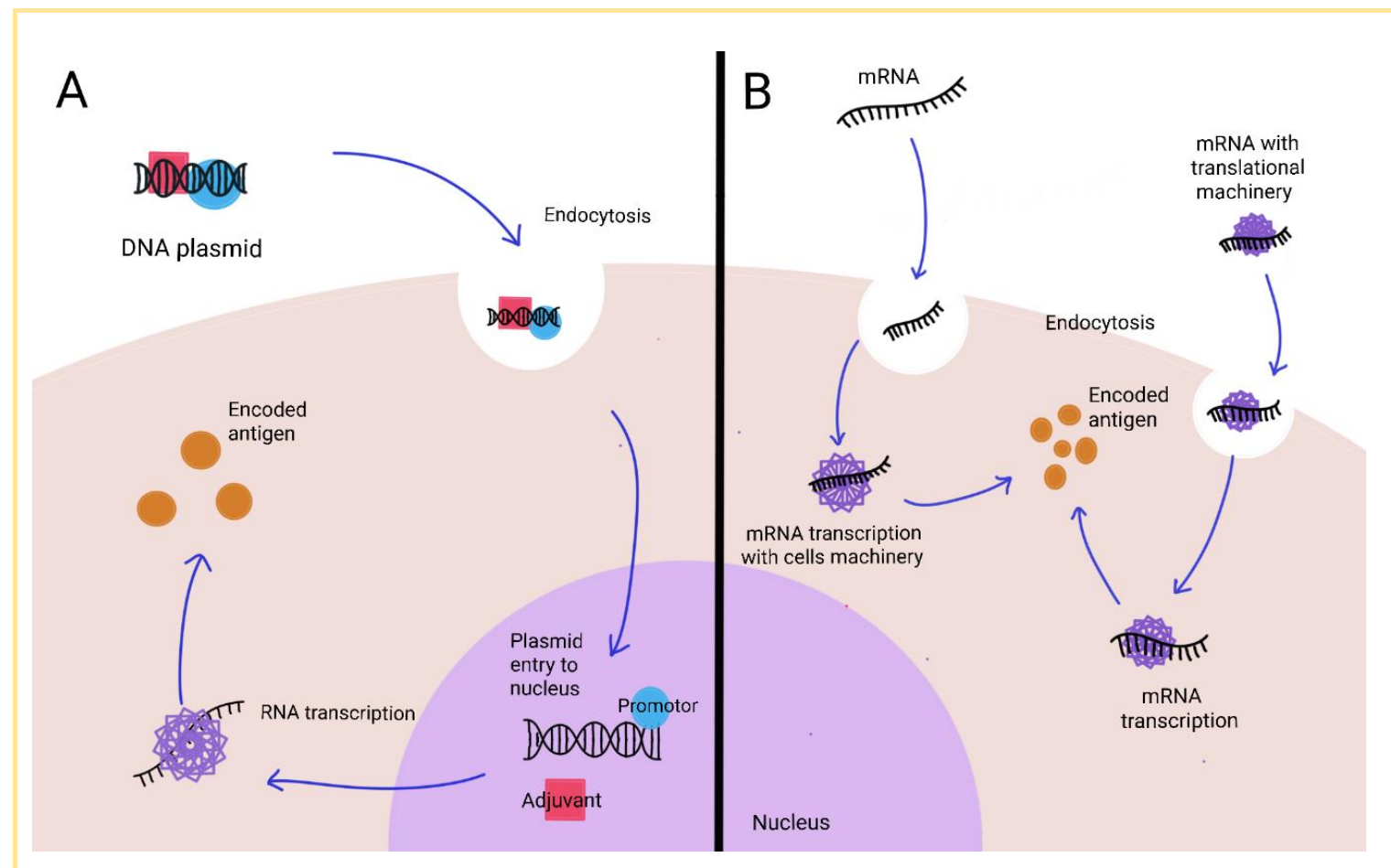

Figure 2. Nucleic acid vaccines. A. A bacterial plasmid is transfected with an antigen gen flanked by a mammalian promoter. Disregarding the route of administration, after plasmid endocytosis, the genetic material reaches nucleus cells and begins RNA transcription, thus antigen is synthetized. B. Conventional mRNA vaccines need cells transcription machinery to encode the antigen, meanwhile self-amplifying RNA encode the antigen and the transcription machinery.

Once the DNA plasmid is in the nucleus, transfected cells initiate transcription and translation to encode the protein of interest, mediated by a mammalian promoter (Fig. 2). Then, the antigen is expressed by the host's APCs, through the MHC class I and/or II molecules to activate naïve T cells. Stromal cells (e.g. myocytes) may act also as APCs through the MHC-I, allowing antigen to be cross-presented to CD8 $8^{+} \mathrm{T}$ cells. MHC-I pathway activates CD8 ${ }^{+} \mathrm{T}$ cell to induce cytokine release (e.g. tumoral-necrosis factor $\alpha[\mathrm{TNF}-\alpha]$ and interferon $\gamma$ [IFN- $\gamma]$ ) to decrease viral replication, induce cellular apoptosis mediated by degranulation, and lead more MHC-I expression. On the other hand, MHC-II pathway activates CD $4^{+} \mathrm{T}$ cells to release cytokines to stimulate other immune cell responses. Additionally, poured antigens after cell apoptosis can be recognized by immunoglobulins on B cell surface, leading humoral response, with their activation to plasmatic cells (PC) that will produce antibodies against the pathogen 26,27,29.

\section{RNA VACCINES}

Nucleic acid vaccines appear as a promising option to replace traditional methods. Indeed, mRNA technology confers superiority even to DNA vaccines. There are two major groups of RNA vaccines: conventional non-replicating mRNA that encode only the antigen; and self-amplifying RNA, that encodes the antigen and the viral genetic replication machinery (excluding the capsid, proteases and other 
viral genes) (Fig. 2). The mRNA is protected by transfection reagents to prevent degradation and to facilitate cellular uptake. As such, they are composed by an open reading-frame that codifies the disease-specific antigen surrounded by a guanosine cap at the 5' end (5'cap) and a polyadenylated tail (polyA). This structure leads increased antigen output by augmenting translation and/or vector persistence ${ }^{31,32}$.

Inside the cell, the translational machinery produces an entirely functional viral protein based on the vaccine's mRNA ${ }^{30}$. In terms of safety, RNA vaccines are noninfectious because they do not include the entire pathogen, not the complete set of genes to express its whole products. Additionally, there is no interaction between the mRNA and the host's DNA, so that genome mutations are not expected ${ }^{32}$. Finally, after the protein is expressed, mRNA is degraded by physiological mechanisms, which reduce possibilities of metabolite toxicity ${ }^{30}$.

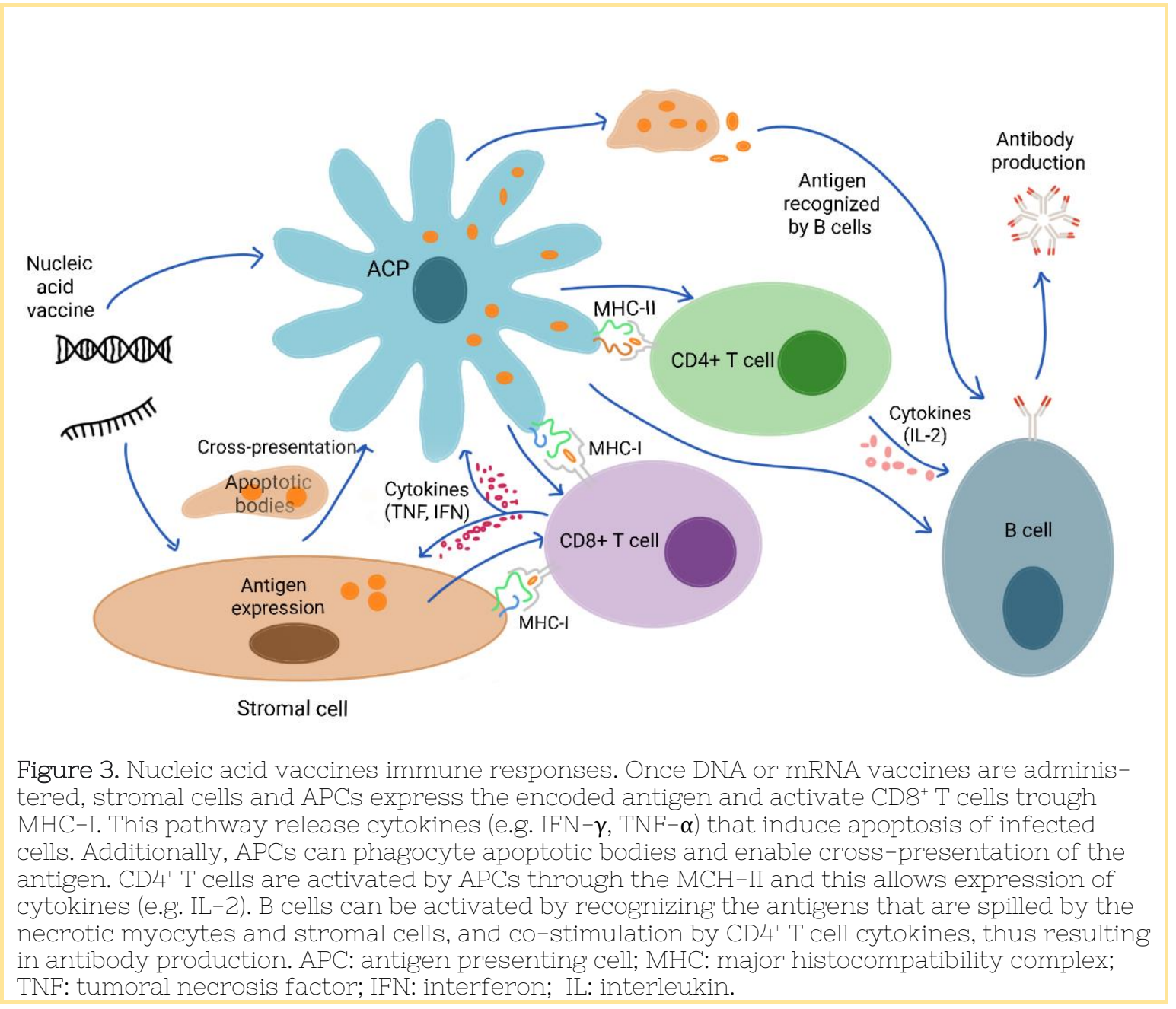

mRNA vaccines are able to induce efficient CD8 ${ }^{+} \mathrm{T}$ cell responses, due to the presentation of antigens on MHC-I molecules, which is more effective than immunization with protein agents. Moreover, the immunity is complimented by a 
SÖC̊IIÉÉÁAD

ESPAÑOLA BENEFICENCIA $A C$

P A $\quad$ C

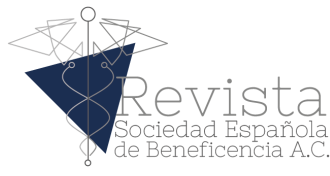

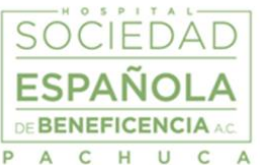

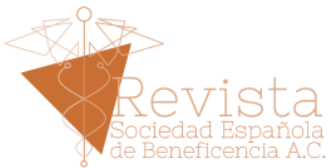

strong $\mathrm{CD}^{4}{ }^{+} \mathrm{T}$ cell response, and both effects can be possible with only one or two doses, as shown in animal models ${ }^{30}$. Finally, the humoral response to such vaccines occur when CD8 ${ }^{+} \mathrm{T}$ cells induce the necrosis of the transfected cells, in which the expressed antigens are spilled on the extracellular space and carried through the lymphatic system.

Additionally, B and T cell response seems to be influenced by other processes, such as the antigen availability time, the participation of other cells of the innate immune system, and the cytokine release induced by both delivery compounds and mRNA in the vaccine Thus, a good way to evaluate the activity of mRNA vaccines front specific antigens is measuring B and T cell responses ${ }^{31}$. As mentioned, the potential for this technology to replace traditional immunization methods has been seen in the COVID-19 pandemic, where a fast development, approval and production of mRNA vaccines occurred.

\section{Conclusions}

Vaccines are the most cost-effective measure to control infectious diseases. Traditional vaccination methods require longer production times and higher costs for development than the newer vaccine technologies. In this context nucleic acid vaccines appear as a promising alternative, nonetheless, viral vector-driven vaccines may also be good candidates, as they promote an immune response that is nearly identical to that of the naturally acquired infection, and thus it may function in an equivalent way. These vaccines also require less time for production and approval, and their thermostability allow for their availability in any region. Additionally, these technologies seem to be safe. All these data together suggests that the viral vector and nucleic acid vaccine formulations have a great potential to promptly respond to emerging diseases, but are not only limited to them.

\section{References}

1 Graham, B. S. \& Sullivan, N. J. Emerging viral diseases from a vaccinology perspective: preparing for the next pandemic. Nat Immunol 19, 20-28, doi:10.1038/s41590-017-0007-9 (2018).

2 Dube, E. et al. Vaccine hesitancy: an overview. Hum Vaccin Immunother 9, 1763-1773, doi:10.4161/hv.24657 (2013).

3 Durán-Méndez, A., Jardínez-Vera, A. C., Jiménez-Muñoz, E. \& Peón, A. N. A brief history of vaccines and an overview of their benefits. Revista de la Sociedad Española de Beneficencia 2, 6, doi:https://doi.org/10.46295/2:1.VACHI (2021).

4 Rauch, S., Jasny, E., Schmidt, K. E. \& Petsch, B. New Vaccine Technologies to Combat Outbreak Situations. Front Immunol 9, 1963, doi:10.3389/fimmu.2018.01963 (2018).

5 Crommelin, D. J. A., Volkin, D. B., Hoogendoorn, K. H., Lubiniecki, A. S. \& Jiskoot, W. The Science is There: Key Considerations for Stabilizing Viral Vector- 
Based Covid-19 Vaccines. J Pharm Sci 110, 627-634, doi:10.1016/j.xphs.2020.11.015 (2021).

6 Noriega-Rubalcaba, A. et al. Estrategia de vacunación en México y diversidad de vacunas. Revista de la Sociedad Española de Beneficencia 2, 11, doi: https://doi.org/10.46295/2:1.CORREC (2021).

$7 \quad$ Sandbrink, J. B. \& Shattock, R. J. RNA Vaccines: A Suitable Platform for Tackling Emerging Pandemics? Front Immunol 11, 608460, doi:10.3389/fimmu.2020.608460 (2020).

8 Amanpour, S. The Rapid Development and Early Success of Covid 19 Vaccines Have Raised Hopes for Accelerating the Cancer Treatment Mechanism. Arch Razi Inst76, 1-6, doi:10.22092/ari.2021.353761.1612 (2021).

9 Golob, J. L., Lugogo, N., Lauring, A. S. \& Lok, A. S. SARS-CoV-2 vaccines: a triumph of science and collaboration. JCI Insight, doi:10.1172/jci.insight.149187 (2021).

10 Kuter, B. J., Offit, P. A. \& Poland, G. A. The development of COVID-19 vaccines in the United States: Why and how so fast? Vaccine, doi:10.1016/j.vaccine.2021.03.077 (2021).

11 Verbeke, R., Lentacker, I., De Smedt, S. C. \& Dewitte, H. Three decades of messenger RNA vaccine development. Nano Today 28, 100766, doi:https://doi.org/10.1016/j.nantod.2019.100766 (2019).

12 Brisse, M., Vrba, S. M., Kirk, N., Liang, Y. \& Ly, H. Emerging Concepts and Technologies in Vaccine Development. Front Immunol 11, 583077, doi:10.3389/fimmu.2020.583077 (2020).

13 Plotkin, S. A. Vaccines: the fourth century. Clin Vaccine Immunol 16, 17091719, doi:10.1128/CVI.00290-09 (2009).

14 Shirley, J. L., de Jong, Y. P., Terhorst, C. \& Herzog, R. W. Immune Responses to Viral Gene Therapy Vectors. Mol Ther 28, 709-722, doi:10.1016/j.ymthe.2020.01.001 (2020).

15 Afrough, B., Dowall, S. \& Hewson, R. Emerging viruses and current strategies for vaccine intervention. Clinical and experimental immunology 196, 157-166, doi:10.1111/cei.13295 (2019).

16 Bulcha, J. T., Wang, Y., Ma, H., Tai, P. W. L. \& Gao, G. Viral vector platforms within the gene therapy landscape. Signal transduction and targeted therapy 6 , 53, doi:10.1038/s41392-021-00487-6 (2021).

17 Ewer, K. J. et al. Viral vectors as vaccine platforms: from immunogenicity to impact. Current opinion in immunology 41, 47-54, doi:10.1016/j.coi.2016.05.014 (2016). 
18 Lukashev, A. N. \& Zamyatnin, A. A., Jr. Viral Vectors for Gene Therapy: Current State and Clinical Perspectives. Biochemistry. Biokhimiia 81, 700-708, doi:10.1134/s0006297916070063 (2016).

19 Pediatrics, A. A. o. Red Book: 2003 Report of the Committee on Infectious Diseases 26 edn, (American Academy of Pediatrics, 2003).

20 Delrue, I., Verzele, D., Madder, A. \& Nauwynck, H. J. Inactivated virus vaccines from chemistry to prophylaxis: merits, risks and challenges. Expert Rev Vaccines 11, 695-719, doi:10.1586/erv.12.38 (2012).

21 Kitching, R. P. Vaccines for lumpy skin disease, sheep pox and goat pox. Developments in biologicals 114, 161-167 (2003).

22 Abdelwahab, M., Khafagy, H., Moustafa, A. \& Saad, M. Evaluation of Humoral and Cell-mediated Immunity of Lumpy Skin Disease Vaccine Prepared from Local strainin calves and Its Related to Maternal Immunity Evaluation of Humoral and Cell-mediated Immunity of Lumpy Skin Disease Vaccine Prepared from Local in calves and Its. Journal of American Science, doi:10.7537/marsjas121016.06 (2016).

23 Barrett, P. N., Terpening, S. J., Snow, D., Cobb, R. R. \& Kistner, O. Vero cell technology for rapid development of inactivated whole virus vaccines for emerging viral diseases. Expert Review of Vaccines 16, 883-894, doi:10.1080/14760584.2017.1357471 (2017).

24 Chen, R. T. \& Orenstein, W. A. Epidemiologic methods in immunization programs. Epidemiologic reviews 18, 99-117, doi:10.1093/oxfordjournals.epirev.a017931 (1996).

25 Li, L. \& Petrovsky, N. Molecular mechanisms for enhanced DNA vaccine immunogenicity. Expert review of vaccines 15, 313-329, doi:10.1586/14760584.2016.1124762 (2016).

26 Suschak, J. J., Williams, J. A. \& Schmaljohn, C. S. Advancements in DNA vaccine vectors, non-mechanical delivery methods, and molecular adjuvants to increase immunogenicity. Hum Vaccin Immunother 13, 2837-2848, doi:10.1080/21645515.2017.1330236 (2017).

27 Silveira, M. M., Moreira, G. \& Mendonça, M. DNA vaccines against COVID19: Perspectives and challenges. Life sciences 267, 118919, doi:10.1016/j.lf.s.2020.118919 (2021).

28 Bai, H., Lester, G. M. S., Petishnok, Laura C. \& Dean, David A. Cytoplasmic transport and nuclear import of plasmid DNA. Bioscience Reports 37, doi:10.1042/BSR20160616 (2017).

29 Coban, C., Kobiyama, K., Jounai, N., Tozuka, M. \& Ishii, K. J. DNA vaccines. Human Vaccines \& Immunotherapeutics 9, 2216-2221, doi:10.4161/hv.25893 (2013). 


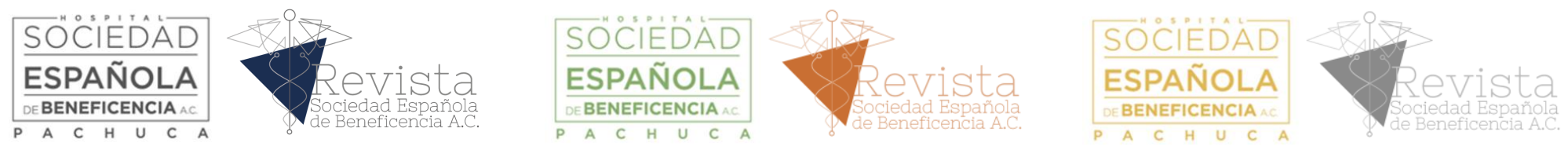

30 Pardi, N., Hogan, M. J., Porter, F. W. \& Weissman, D. mRNA vaccines - a new era in vaccinology. Nature Reviews Drug Discovery 17, 261-279, doi:10.1038/nrd.2017.243 (2018).

31 Kramps, T. \& Elbers, K. Introduction to RNA Vaccines. Methods in molecular biology (Clifton, N.J.) 1499, 1-11, doi:10.1007/978-1-4939-6481-9_1 (2017).

32 Lin, C. J., Mecham, R. P. \& Mann, D. L. RNA Vaccines for COVID-19: 5 Things Every Cardiologist Should Know. JACC. Basic to translational science 5, 1240-1243, doi:10.1016/j.jacbts.2020.11.006 (2020). 